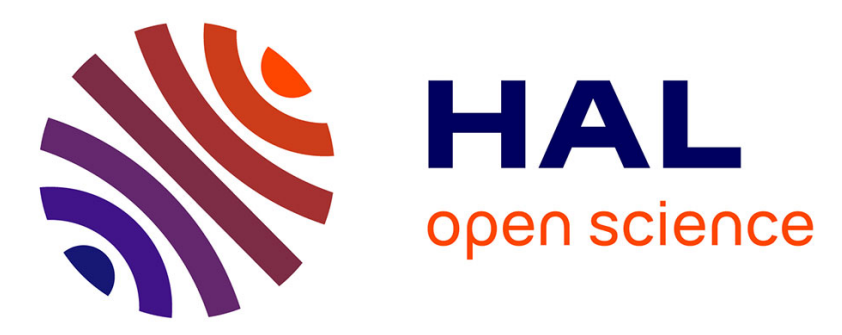

\title{
Autonomous configuration control for UAV formation flight in hostile environments
}

Gautier Hattenberger, Rachid Alami, Simon Lacroix

\section{To cite this version:}

Gautier Hattenberger, Rachid Alami, Simon Lacroix. Autonomous configuration control for UAV formation flight in hostile environments. IFAC Proceedings Volumes, 2007, 40 (15), pp.451-456. 10.3182/20070903-3-FR-2921.00077 . hal-01979770

HAL Id: hal-01979770

https://hal.laas.fr/hal-01979770

Submitted on 13 Jan 2019

HAL is a multi-disciplinary open access archive for the deposit and dissemination of scientific research documents, whether they are published or not. The documents may come from teaching and research institutions in France or abroad, or from public or private research centers.
L'archive ouverte pluridisciplinaire HAL, est destinée au dépôt et à la diffusion de documents scientifiques de niveau recherche, publiés ou non, émanant des établissements d'enseignement et de recherche français ou étrangers, des laboratoires publics ou privés. 


\title{
AUTONOMOUS CONFIGURATION CONTROL FOR UAV FORMATION FLIGHT IN HOSTILE ENVIRONMENTS
}

\author{
Gautier Hattenberger ${ }^{* 1}$ Rachid Alami ${ }^{*}$ \\ Simon Lacroix* \\ * LAAS - CNRS, University of Toulouse \\ Toulouse, France
}

\begin{abstract}
A fleet of UAVs flying according a planned mission in hostile environments must optimize its configuration, so that the UAVs auto-protection systems ensure the fleet safety. Such an optimization can be done in the mission planning phase, and must also be reactively updated to tackle unpredicted threats. In this paper, we present an approach to the problem of selecting autonomously the fleet configuration. Algorithms that explicitly consider models of the threats and of the countermeasures systems are presented, and integrated within a global decisional architecture that ensures reactiveness and constraints satisfactions.
\end{abstract}

Keywords: Autonomous vehicles, Aircraft operations, Trajectory planning, Potentials, Slot assignment algorithms

\section{INTRODUCTION}

The control of multiple-UAVs systems offers many challenging topics for both theoretical and practical research. Since aerial vehicles can be used in a wide range of applications, such as exploration or surveillance, in military or civilian scenarios, each case of study is a specific problem and may not be treated as global. This paper is focused on the management of UAVs flying in tight coordination, i.e. in formation flight, in a hostile environment. The formation flight is indeed very suited for applications that requires synchronizations on target arrivals or mutual support, typically in defense applications. One of the difficulties raised in such cases is the autonomous choice of a configuration for the formation that improve the safety and the efficiency of the team of UAVs.

\footnotetext{
1 The authors would like to thank Dassault Aviation for their support to this work.
}

Related work. Most of the work related to the formation flight problem are focused on the control of the formation itself. Many solutions have been presented based on proportional-integral control (Buzogany et al., 1993) or non-linear control (Schumacher and Singh, 2000). They often deal with classical leader-wingman configuration and they usually do not tackle the problem of the choice of the configuration. The behavior based control (Balch and Arkin, 1998) have shown some capacities for handling basic reconfigurations of holonomic robots moving in formation. Algorithms for trajectory optimization have been presented with a centralized (Lian and Murray, 2002) or a distributed solution (Raffard et al., 2004). Nevertheless, some contributions have been proposed on the configuration of the formation itself (Giulietti et al., 2000) or on the reconfiguration problem. The work of (Zelinski et al., 2003) is based on trajectories computed off-line between a finite number of configurations. On the other side, the contributions on control architectures dedicated to mission planning for teams of UAVs 
do not consider the choice of the configuration for the formation (Sousa et al., 2004; Ollero et $a l ., 2005)$. It is clear that the two issues, control and mission planning, are tackled separately and that the choice of the configuration should be considered (Chen and Wang, 2005).

Contribution and outline. This work is focused on the autonomous adaptability of the configuration of a team of UAVs flying in formation. A configuration is defined by a set of slots corresponding to the relative position of the aircrafts. The mission is defined by a list of waypoints and a set of tactical constraints. The algorithms lies in an intermediate layer between the mission planning system and the autopilot of each UAV. This approach is motivated by the fact that the deliberative level is released from "internal" formation problems and so, can manipulate the team as a whole. Our layer is in charge of the following functionalities: (1) the choice of a configuration according to the constraints and the environment, (2) the planning of reconfiguration trajectories for safe transitions between two configurations, (3) the flight control loop to achieve the coordination inside the formation. The last two points have been presented in a previous work (Hattenberger et al., 2006), and this paper focuses on the first functionality.

The next section precisely states the problem and describes the elements we consider to solve it. Then, section 3 presents the approach to solve each part of the problem. Section 4 depicts some algorithms involved in UAVs' tasks and slots allocation. Finally, section 5 shows the global update process of the formation's configuration and its integration within the control architecture. Some perspectives and discussions conclude the paper.

\section{PROBLEM STATEMENT}

\subsection{Mission definition}

A group of at most eight aircrafts flying in formation is considered. It is a realistic value for military operations considering the payload and the stealthiness of a group of planes. It is assume that a mission planning phase has been carried out either off-line or re-planned on-line. The outputs of this phase are an ordered list of waypoints, a set of tactical constraints and a set of known threats. Waypoints are produced by the deliberative layer and can't be changed by the formation layer. The trajectory generated from these waypoints is a succession of straight line and circle's arcs. It is a reference for a virtual center in the formation and not the actual trajectory of the UAVs.

\subsection{Threats jamming model}

Two types of threats are considered. Early Warning $(E W)$ radars and missiles (Track \& Fire, $T F$ ).

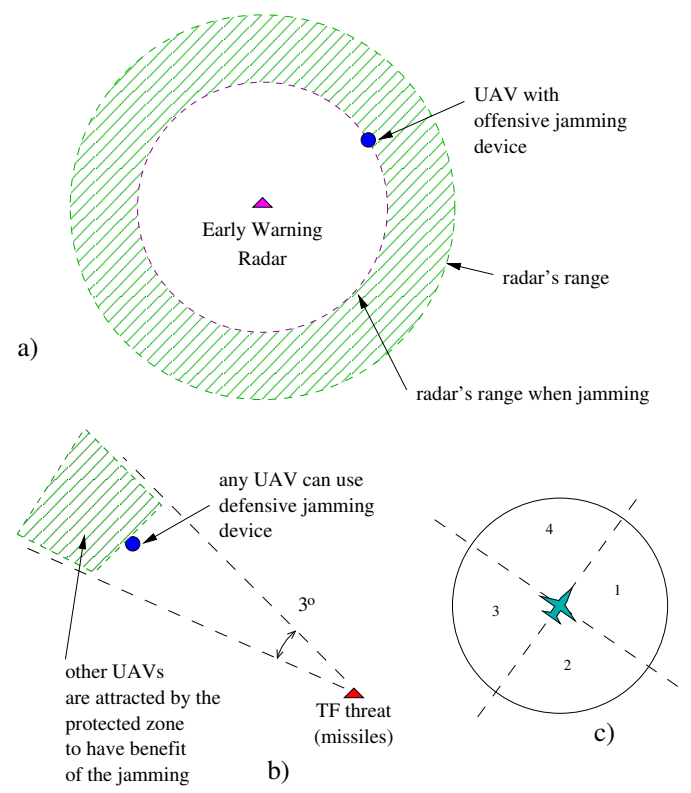

Fig. 1. a) Effects of offensive jamming on $E W$ radars. b) Defensive jamming on $T F$ threats. c) Four sectors defined for the UAVs.

Early Warning $(E W)$ are radars used for detection. They control several missiles sites located in a given range around the radar position. The position of the radar is usually known during the initial off-line planning phase since they are permanently turned on. This kind of radar can be jammed using a specific device, which is the only payload that can be carried by one aircraft. These aircrafts will be referred as "offensive jammers" $(O J)$. The effect of this device is to reduce the range of the radar, so that any aircrafts at a larger distance from the $E W$ radar than the jammer will not be detected (see Fig. 1.a).

Track 8 Fire $(T F)$ systems are sol to air missiles sites controlled by $E W$ radars. They usually have reactive behaviors and their position can't be well known by advance. If they use missiles guided by a radar, the threat can be jammed using a light protection device called "defensive jammer" $(D J)$. The effect is to blind the guiding system of the $T F$ threat in the direction of the jammer in a cone of aperture of $3^{\circ}$ (see Fig. 1.b). All aircrafts behind the jammer inside this cone will benefit of the protection. If infra-red missiles are used, the threat cannot be jammed.

For each UAV, sectors are defined as shown Fig. 1.c. Three $E W$ threats can be jammed inside each sector with an $O J$, and a single $T F$ threat can be jammed by sector with a $D J$. 


\subsection{Constraints}

Two kind of constraints hold between two given waypoints:

Internal constraints are relative to the inner organization of the formation. A minimum and maximum distance between the UAVs ensure a secured flight and a good communication level. A geometric configuration can be specified manually, and for each slot inside the formation, the type of UAV allowed on this position.

External constraints are caused by the context. An important one is the width of the corridor in which the UAVs must fly. For each threat, it is possible to explicitly define if it should be taken into account (with a higher piority) or not. Formation split and join maneuvers are considered as external constraints, and a distribution of payload can be specified.

\section{GLOBAL APPROACH}

\subsection{Formation update}

The approach is first to initiate the system by computing the $O J$ allocation and the timeline. Then, at each step the configuration of the formation is updated based on the allocation, the current interval in timeline, the detected $T F$ threats and the position of the formation on the trajectory. The system is also updated when constraints, threats or waypoints are added or removed. Some of these updates can lead to a new initialization of the $O J$ allocation or the timeline. Otherwise, the new values will be taken into account at the next iteration. Fig. 2 summarize this process.

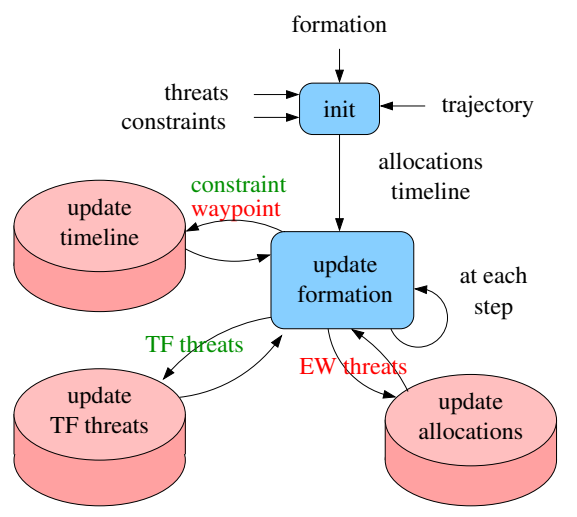

Fig. 2. The global update process. Red elements require a new initialization or a part of it, while green elements do not.

\subsection{Motion modes}

As mentioned in section 2.2, EW and $T F$ threats have different behaviors and the system knowledge on these threats is not the same either. So, a different control of the configuration should be chosen according to the kind of threats to be treated. Three modes are defined:

reactive mode is a behavior based motion mode using a potential field associated with the $T F$ threats. The forces are designed to grant mutual support for the UAVs when they are using their $D J$. It is also the default motion mode.

parametric mode is used to place the $O J$ in the right position in order to create the best protection for the rest of the formation. This position is determined according to the knowledge of the $E W$ threats and the $O J$ allocation. This mode is combined with the reactive mode.

planned mode is used to execute reconfiguration trajectories. They are computed when important changes occur in the formation (positions or slots' allocation). This mode takes control over the two others.

\subsection{The timeline}

It is mentioned in section 2.3 that the constraints are defined between two waypoints. The timeline is a structured representation of the set of constraints along the trajectory. It is composed of intervals that contain the most restrictive information for each type of constraints as shown Fig. 3. The parameters for configuration selection and reconfiguration trajectories computation are taken from the current interval in the timeline.

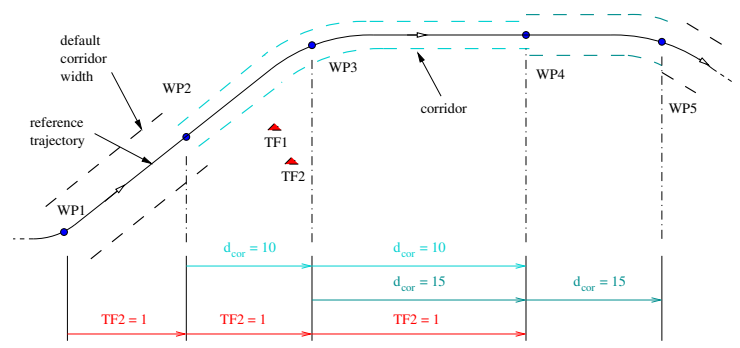

Fig. 3. Example of timeline with 3 constraints. Two constraints on the corridor width and one that gives higher priority to threat TF2/

\subsection{Offensive jammers allocation}

For $O J$ allocation, the $E W$ threats' position and range, the trajectory and the sectors constraints (see section 2.2) are considered. An allocation $A$ is defined for one jammer as a set of threats in the range of the formation, that respects the limitation on sectors, maximum aperture and that are connex (see Fig. 4). If there is only one threat, the aperture is zero and the protection is maximal. The maximum aperture is $180^{\circ}$ and the protection is minimal. So, the quality criterion $Q$ 
for an allocation $A$ is defined as the ratio between the aperture and the number of threats in $A$. The global $O J$ allocation algorithm (section 4.1) finds the optimal set of allocations $A$ that minimize the sum of $Q$ with the minimum number of jammers.

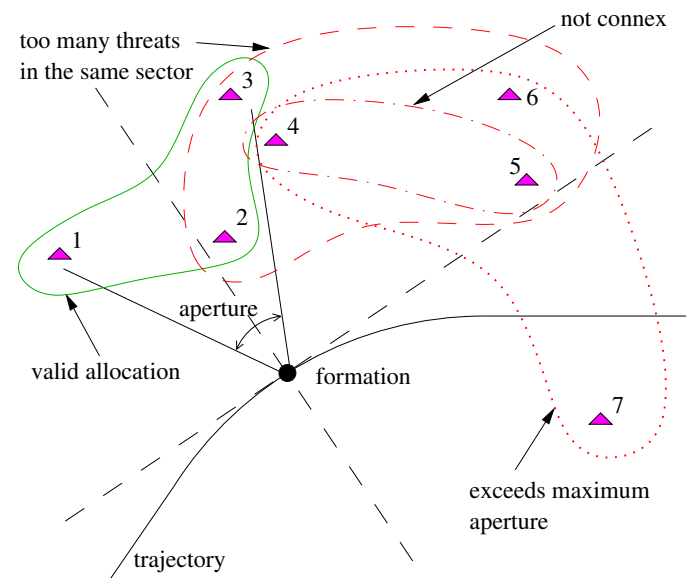

Fig. 4. Example of valid (green) and not valid (red) allocations for a maximum aperture of 90 degrees.

\section{ALGORITHMS}

\subsection{Jammer allocation}

The $O J$ allocation process is based on a Branch E Bound algorithm. For each step on a dicretized trajectory, we want to find the set of allocations $A_{i}$ with quality $Q_{i}$ that minimize the cost $C=N+$ $\sum_{i=1}^{N} Q_{i}$, where $N$ is the number of allocations. The algorithm is initialized with a graph that contains all the possible allocations without doublet. Table 1 shows an example of graph initialized with the situation shown on Fig 4.

Table 1.

\begin{tabular}{cll}
\hline Threat & \multicolumn{2}{l}{ Associated allocations } \\
\hline 1 & - & $(1)(1,2)(1,2,3)(1,2,3,4)$ \\
2 & - & $(2)(2,3)(2,3,4)(2,3,5,6)$ \\
3 & - & $(3)(3,4)(3,4,6)(3,4,6,5)$ \\
4 & - & $(4)(4,6)(4,6,5)$ \\
5 & - & $(5)(6,5)(5,7)(6,5,7)$ \\
6 & - & $(6)$ \\
7 & - & \\
\hline
\end{tabular}

At each iteration, the allocation $A$ with the best quality $Q$ is picked. Assuming that $A$ is associated with the threat $T$, two choices are made: " $A$ is part of the global allocation" and " $A$ is not part of the global allocation". In the first case, $A$ is added to the solution and the graph is updated by removing all the allocations where the threats in $A$ appears. The cost $C$ is computed and the bound $B$ is equal to it. In the second case, $A$ is removed from the graph. The cost $C$ doesn't change and the bound $B=C+1$ states that to find a solution from this node, one extra allocation is at least needed. The next step starts from the most promising node (with the lowest bound). If this node is a solution, it is the optimal one.

\subsection{Parametric and reactive moves}

During the update process of the configuration, if not in planned mode, the first slots to be set are those in parametric mode. The number of parametric slots is the number of allocations and can not exceed the number of $O J$. Considering an allocation, the slot is on the bisecting line of the angle that defines its aperture. The distance $d_{p}$ between the slot and the center of the formation is automatically set according to the formula:

$$
d_{p}=d_{c o r}\left(1-e^{-\frac{d_{\min (n-1)}}{d_{c o r}}}\right)
$$

where $d_{\text {cor }}$ is the corridor width, $n$ the number of UAVs and $d_{\text {min }}$ the minimum security distance between aircrafts. If $d_{\text {min }}$ is close to $d_{c o r}$, the protected space for the formation is bigger. The need of protected space will increase faster if the ratio $d_{\text {cor }} / d_{\text {min }}$ is small. In order to provide a smooth transition between two different allocations of the same $O J$, the position of the slot can be a weighted sum of the current position and the next one.

Once a valid configuration has been set according to the constraints, the slots in reactive mode move according to a potential field. The forces of this field provide a repulsion from the border of the corridor (equ. 2), from the other UAVs (3) and from the areas not protected by $O J(4)$. For an UAV $i$ at position $\boldsymbol{x}_{\boldsymbol{i}}$ in the local reference frame of the formation:

$$
\begin{gathered}
\boldsymbol{f} \boldsymbol{c}_{\boldsymbol{i}}=\frac{-1}{\left(\left\|\boldsymbol{x}_{\boldsymbol{i}}\right\|-d_{\text {cor }}\right)^{2}} \frac{\boldsymbol{x}_{\boldsymbol{i}}}{\left\|\boldsymbol{x}_{\boldsymbol{i}}\right\|} \\
\boldsymbol{f} \boldsymbol{u}_{\boldsymbol{i}}=\sum_{\substack{j \in F \\
j \neq i}} \frac{1}{\left(\left\|\boldsymbol{x}_{\boldsymbol{i}}-\boldsymbol{x}_{\boldsymbol{j}}\right\|-d_{\text {min }}\right)^{2}} \frac{\boldsymbol{x}_{\boldsymbol{i}}-\boldsymbol{x}_{\boldsymbol{j}}}{\left\|\boldsymbol{x}_{\boldsymbol{i}}-\boldsymbol{x}_{\boldsymbol{j}}\right\|} \\
\boldsymbol{f} \boldsymbol{t}_{\boldsymbol{i}}=\sum_{t \in T} \frac{1}{\left(\left\|\boldsymbol{x}_{\boldsymbol{i}}-\boldsymbol{x}_{\boldsymbol{t}}\right\|-r_{t}\right)^{2}} \frac{\boldsymbol{x}_{\boldsymbol{i}}-\boldsymbol{x}_{\boldsymbol{t}}}{\left\|\boldsymbol{x}_{\boldsymbol{i}}-\boldsymbol{x}_{\boldsymbol{t}}\right\|}
\end{gathered}
$$

where $r_{t}$ in (4) is the jammed range of an $E W$ threat, $F$ in (3) is the set of UAVs, $T$ in (4) is the set of jammed $E W$ threats. The last force (7) is an attraction from the areas protected by $D J$ :

$$
\begin{aligned}
\boldsymbol{m}_{\boldsymbol{i j}}=\sum_{k \in S_{j}} \begin{cases}-\left(\boldsymbol{v} \cdot \boldsymbol{w}_{\perp}\right) e^{-\boldsymbol{v} \cdot \boldsymbol{w}_{1}} \boldsymbol{w}_{\perp} & \text { if } \boldsymbol{v} \cdot \boldsymbol{w}>0 \\
\|\boldsymbol{v}\| e^{\boldsymbol{v} \cdot \boldsymbol{w}} \boldsymbol{v} & \text { otherwise }\end{cases} \\
\text { with } \| \begin{array}{l}
\boldsymbol{w}=\frac{\boldsymbol{x}_{\boldsymbol{j}}-\boldsymbol{x}_{\boldsymbol{k}}}{\left\|\boldsymbol{x}_{\boldsymbol{j}}-\boldsymbol{x}_{\boldsymbol{k}}\right\|} \\
\boldsymbol{w}_{\perp} \text { orthogonal to } \boldsymbol{w} \\
\boldsymbol{v}=\boldsymbol{x}_{\boldsymbol{i}}-\boldsymbol{x}_{\boldsymbol{j}}+d_{\text {min }} \cdot \boldsymbol{w}
\end{array} \\
\boldsymbol{f}_{\boldsymbol{i}}=\sum_{j \in R} \boldsymbol{m}_{\boldsymbol{i j}}+\sum_{l \in P}\left(\boldsymbol{m}_{\boldsymbol{i l}}+\boldsymbol{m}_{\boldsymbol{l i}}\right)
\end{aligned}
$$




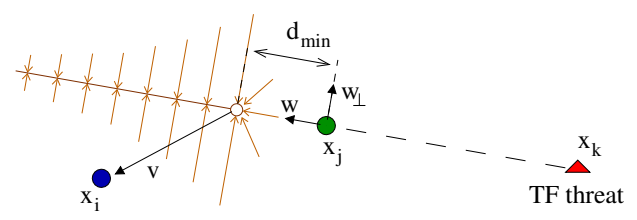

Fig. 5. Attraction force for defensive jamming. The shape of the potential field appears in brown. An UAV is attracted by a "line" of potential if it is behind the jammer.

where $S_{j}$ in (5) is the set of $T F$ threats jammed by UAV $j, R$ and $P$ in (7) are respectively the set UAVs in reactive mode and the one in parametric mode. Convention for equation (6) are shown Fig. 5. The global force applied to a slot is a weighted sum of (2), (3), (4) and (7): $\boldsymbol{f}_{\boldsymbol{i}}=\alpha_{1} . \boldsymbol{f}_{\boldsymbol{c}}+$ $\alpha_{2} \cdot \boldsymbol{f}_{\boldsymbol{u}}+\alpha_{3} \cdot \boldsymbol{f}_{\boldsymbol{t}}+\alpha_{4} \cdot \boldsymbol{f}_{\boldsymbol{a}}$.

The set $S_{j}$ is built as follows: for each TF threat, the closest slot whose $D J$ is free in the appropriate sector is selected as jammer and the slots in the protected area are put aside. We iterate with the remaining slots until they are all checked. It is possible that some slots can't be protected. Moreover, a potential fields can lead to a local minima, but this will not endanger the formation since a valid configuration is always provided.

Fig. 6 shows some examples of these forces. Concerning attraction forces, slot 4 is attracted by the center of the green zone, so it can have a better protection from 5 . Slot 2 is not protected by 5 , but as it is in parametric mode, it is not influenced by the potential field but it has an effect (second part of equation (7)) on the other slots. Concerning $O J$ in green, the unprotected area produced by 1 with allocation $(1,2,3,4)$ is smaller than the one of 2 . This is due to the fact that the aperture of the first allocation is smaller than the second one.

\section{GLOBAL SCHEME}

\subsection{Update process}

In the previous sections, the elements of the problem and some algorithms used to solve it were presented. Here, all the parts are put together and the whole process of update is depicted Fig. 7.

The first step is to compute the $O J$ allocation and the timeline as presented Fig. 2, on the basis on the formation and the reference trajectory. Then, at each step the following sequence is achieved. If the formation is in planned mode, the reconfiguration trajectories are executed until the final configuration is reached or if the planned mode is interrupted to check if a better final configuration can be found. If not in planned move, the parametric slots are updated based on the current allocation, the timeline and the ressources

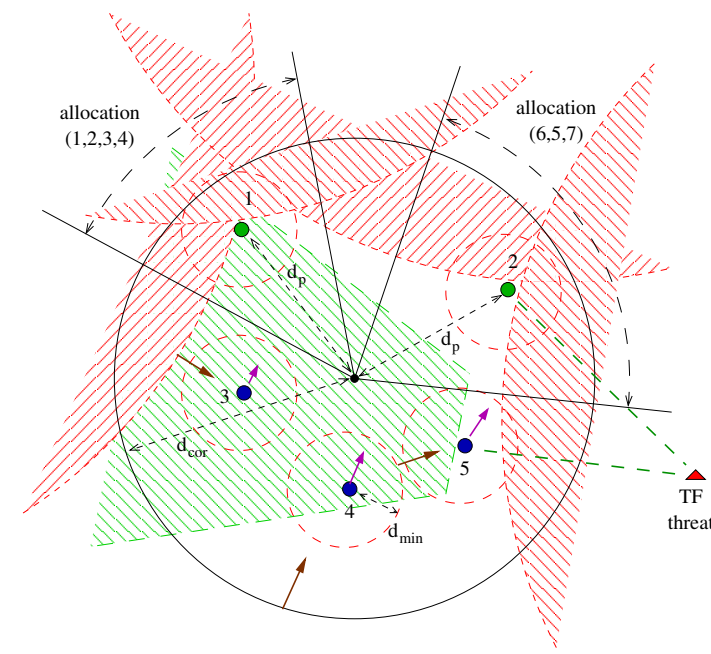

Fig. 6. Example of configuration based on the situation on Fig. 4. Parametric slots are in green, reactive slots in blue, unprotected area $(O J)$ are red-striped, protected area $(D J)$ green-striped, repulsive forces in brown and attractive forces in magenta.

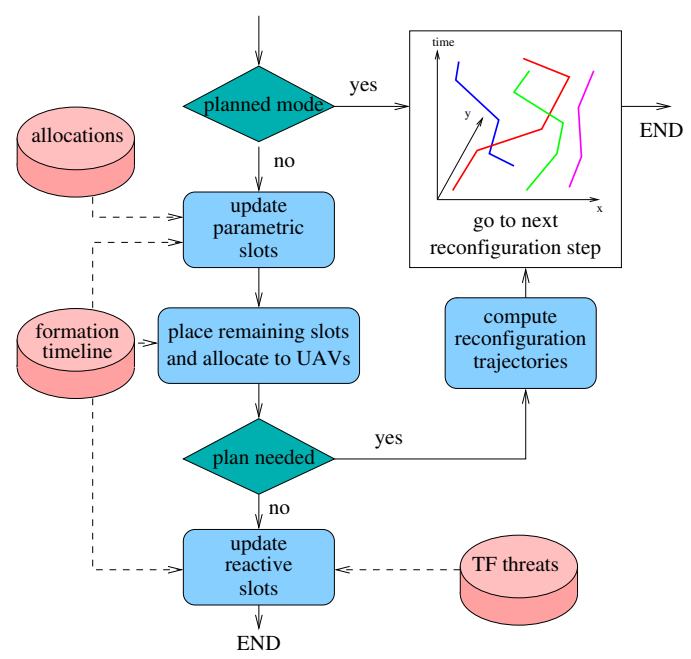

Fig. 7. Formation update process.

of the formation (the number of $O J$ ). Then, the remaining slots are placed and the resulting set of slots is allocated to the UAVs using a Branch $\mathbb{E}$ Bound algorithm (Hattenberger et al., 2006). The next step is to check if a computation of reconfiguration trajectories is needed. If it is the case, an algorithm based on graph search (Hattenberger et al., 2006) is run and its output is executed. Else, the slots in reactive mode move according to the potential field created by the formation and the environment.

\subsection{Architecture}

Organizing the algorithms within the formation is very important, for the sake of efficiency and robustness. The overall architecture designed to support our algorithms is shown Fig. 8. At its top 
level, the deliberative layer receives the missions to achieve from the control center, and produces a mission plan for the formation, as defined in section 2 .

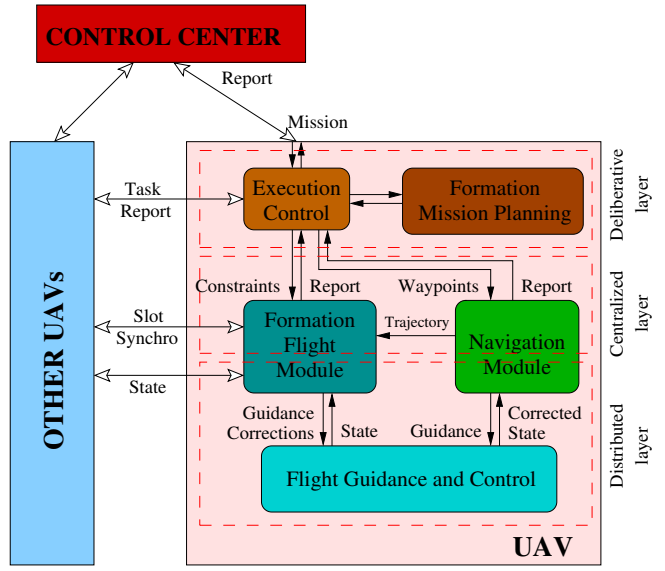

Fig. 8. The global architecture. The formation's configuration update process lies in the Formation Flight Module.

The algorithms involved in the process described above are centralized in the Formation Flight Module. During the flight, only one UAV is in charge of the management of the formation. However, all the aircrafts have the same set of algorithms and can carry out these tasks. On the contrary, the control for formation flight and trajectory tracking needs to be distributed amongst UAVs for a better robustness. This implies that a good communication is required between aircrafts.

\section{SUMMARY AND DISCUSSION}

A set of algorithms and their integration for the autonomous adaptation of the configuration for UAVs in formation flight in hostile environment were presented. This contributes to the filling of the gap between task planning and and execution for teams of aircrafts with an intermediate formation layer.

The system still has some limitations for real-time applications. The solution for the reconfiguration trajectories problem is a fast practical algorithm, but it has no guarantee in computation time, neither in execution time. This problem is overcome by periodically checking if a better solution can be found, which increases the work load of the CPU of the formation leader. The other limitation with this solution is that the protection and mutual support in planned mode is not taken explicitally into account.

Our future work is focused on a realistic simulation framework where the UAVs are simulated on separated computers communicating through the network. Results can be shown in (Hattenberger et al., 2007). An other important issue is how the deliberative layer can use the most efficiently the formation layer.

\section{REFERENCES}

Balch, T. and R. C. Arkin (1998). Behaviorbased formation control for multirobot teams. IEEE Transactions on Robotics and Automation 14(6), 926-939.

Buzogany, L. E., M. Pachter and J. J. D'Azzo (1993). Automated control of aircraft in formation flight. In: AIAA Guidance, Navigation and Control Conference. Monterey, CA. pp. 1349-1370.

Chen, Yang Quan and Zhongmin Wang (2005). Formation control: a review and a new consideration. In: Intelligent Robots and Systems, 2005. (IROS 2005). 2005 IEEE/RSJ International Conference on. pp. 3181-3186.

Giulietti, F., L. Pollini and M. Innocenti (2000). Autonomous formation flight. Control Systems Magazine 20(6), $34-44$.

Hattenberger, G., R. Alami and S. Lacroix (2006). Planning and control for unmanned air vehicle formation flight. In: International Conference on Intelligent Robots and Systems (IROS'06). Beijing, China.

Hattenberger, G., S. Lacroix and R. Alami (2007). Formation flight: evaluation of autonomous configuration control algorithms. Technical report. LAAS-CNRS, http://www.laas.fr/ ghattenb/doc/iros07.pdf, submitted to IROS'07.

Lian, F-L. and R. M. Murray (2002). Real-time trajectory generation for the cooperative path planning of multi-vehicle systems. In: Proceedings of the 41st IEEE Conference on Decision and Control.

Ollero et al. (2005). Multiple eyes in the skies: architecture and perception issues in the comets unmanned air vehicles project. Robotics \& Automation Magazine, IEEE 12(2), 46-57.

Raffard, R. L., C. J. Tomlin and S. P. Boyd (2004). Distributed optimization for cooperative agents: application to formation flight. In: Proceedings 2004 IEEE Conference on Decision and Control. Nassau, Bahamas.

Schumacher, C. and S. N. Singh (2000). Nonlinear control of multiple uav in close-coupled formation flight. In: AIAA Guidance, Navigation and Control Conference. Denver, CO.

Sousa, J., T. Simsek and P. Varaiya (2004). Task planning and execution for uav teams. In: 43rd IEEE Conference on Decision and Control. Vol. 4. pp. $3804-3810$.

Zelinski, S., T. J. Koo and S. Sastry (2003). Hybrid system design for formations of autonomous vehicles. In: 42nd IEEE Conference on Decision and Control, 2003. Vol. 1. 